\title{
Théorie des humeurs et sexualité
}

\author{
FERROLT Y. \\ U.F.R. de Lettres, Université de Lille III, B.P.149, 59653 Villeneuve d'Ascq Cedex \\ Association pour l'Etude de l'Appareil Reproducteur et de la Psychosomatique (EPARP), 49 \\ Rue de la Bassée, 59000 Lille
}

\section{RESUME}

L'ancienne théorie des humeurs a tellement imprégné les esprits de notre société, depuis plus de deux millénaires, qu'elle reste vivante aujourd'hui malgré l'apparition de nouvelles théories scientifiques qui la rendent caduque. La sexualité est l'un des domaines où sa présence est la plus importante. La théorie des humeurs y conditionne les imaginaires et les comportements ; elle sert de fondement aux raisonnements des médecins et des moralistes ; elle est utilisée pour justifier de nombreuses pratiques sociales. Pourtant la découverte des hormones a conduit à proposer une explication bien différente des phénomènes sexuels, et la représentation du corps liée à la théorie des humeurs ne devrait plus être la nôtre.

Mots-clés : théorie des humeurs, sexualité, histoire de la médecine, castration, sexualité des sportifs.

\section{THEORIE DES HUMEURS ET SEXUALITE}

Beaucoup de nos idées reçues sur la sexualité sont en fait fondées sur des théories qui, après avoir régné pendant des siècles, ont été démontées par la science au cours de son évolution. Car le progrès scientifique n'entraîne pas d'office un changement dans les mentalités et les imaginaires. Et si trente pour cent des Français croient encore que le Soleil tourne autour de la Terre, combien plus nombreux seront ceux qui croiront à des explications dépassées de phénomènes intimes et cachés. C'est ainsi que beaucoup de nos contemporains sont persuadés que faire l'amour est une grande dépense d'énergie que l'on ne doit pas multiplier si l'on veut rester performant intellectuellement ou physiquement dans le rythme de la vie quotidienne, comme lorsque l'on veut garder un sperme de qualité pour une éventuelle procréation. Tout cela parce que l'imagination d'un corps composé de liquides en équilibre est toujours vivante.

\section{LA THEORIE DES HUMEURS}

On peut dater d'Hippocrate (Vè siècle avant J.C.) le premier effort de rationalisation dans la réflexion sur la maladie. Les penseurs de son époque rejettent l'idée d'une maladie qui dépendrait de caprices imprévisibles de la divinité et considèrent qu'elle est un événement naturel, qui obéit à des règles, donc qui est un processus accessible à la raison humaine. La maladie alors provient soit de la perturbation de rapports harmonieux entre l'homme et son milieu, soit de la rupture d'un équilibre entre les composantes du corps. Or ce qui peut bouger dans le corps ce sont les liquides, les "humeurs". 
Celles-ci sont au nombre de quatre (le flegme, le sang, la bile jaune, la bile noire), comme les caractéristiques physiques qui les distingueront (le froid, le chaud, le sec et l'humide):

Il y a deux humeurs humides :

- le flegme, qui est froid ;

- le sang, qui est chaud.

Il y a deux humeurs sèches :

- la bile noire, qui est froide ;

- la bile jaune, qui est chaude.

\begin{tabular}{lll}
\hline & Chaud & Froid \\
\hline Sec & Bile jaune & Bile noire \\
Humide & Sang & Flegme \\
\hline
\end{tabular}

Ces humeurs ne sont pas imaginées, mais sont des liquides dont la présence dans le corps humain, ainsi que leurs caractéristiques, découlent de l'observation.

Le sang voit sa présence révélée par les blessures hémorragiques.

Le phlegme, ou flegme, ou lymphe, ou pituite : il peut prendre plusieurs aspects selon son degré de corruption. Eau pure du liquide céphalo-rachidien, après fracture crânienne, il est plus épais quand il s'agit d'un épanchement lymphatique consécutif à un traumatisme du canal thoracique, visqueux dans les régurgitations acides de l'estomac, les glaires intestinales, les pertes blanches ; et enfin boueux quand il infiltre les tissus sous-cutanés.
La bile jaune (en grec : colè) est brûlante dans l'estomac, dans les entrailles. C'est elle que l'on croit voir dans les hématomes à la phase finale, jaunissant la peau. Parfois elle peut envahir tout le corps, colorant la peau et les yeux, se retrouvant dans les vomissements et les selles.

La bile noire, ou atrabile (en grec : mélancholè) est repérée dans la rate (en anglais : spleen) dont l'intérieur se révèle noir à l'examen sur un cadavre, ce que nous expliquons aujourd'hui par la présence de globules rouges détruits.

L'observation porte aussi sur les saisons et leur lien avec la dominance de l'une ou l'autre humeur. En Hiver, l'humidité du temps augmente celle des corps, la pituite devient surabondante, elle "déborde" et doit s'éliminer : on se mouche beaucoup plus, on crache aussi davantage. Au Printemps, la chaleur s'ajoutant aux pluies, fait reculer l'humeur froide et humide au profit de l'humeur chaude et humide, le sang : les corps rougissent et s'échauffent plus facilement, les hémorragies nasales sont plus fréquentes.

L'été, chaud et sec, voit la prédominance de la bile jaune, qui provoque des fièvres, qui colore la peau. L'automne, sec et froid, ramène la bile noire et toutes les mélancolies, les états dépressifs quand les jours et la lumière diminuent (Tableau 1).

Les perturbations liquidiennes possibles sont logiquement les dépôts (ou stases), les déplacements, ainsi que les problèmes d'évacuation, excessive ou insuffisante.

Tableau 1 : Ce tableau se lit ainsi : en hiver, froid et humide, le flegme est maximum, alors qu'il est minimum en été, chaud et sec. Ou : le flegme, froid et humide, est maximum en hiver. Ou : en hiver, les problèmes peuvent venir de l'excès de flegme ou de l'insuffisance de bile jaune, tandis que sang et bile noire sont équilibrés.

\section{Chaud Froid Humide Sec Flegme Sang B. Jaune B. Noire}

\begin{tabular}{|c|c|c|c|c|c|c|c|c|}
\hline Hiver & & + & + & & ++ & + & - & + \\
\hline Printemps & + & & + & & + & ++ & + & - \\
\hline Eté & + & & & + & - & + & ++ & + \\
\hline Automne & & + & & + & + & - & + & ++ \\
\hline
\end{tabular}


Les principes thérapeutiques découlent des perturbations possibles. Le médecin aura pour objectif de :

- déplacer une humeur mal située ;

- expulser ce qui nuit en stagnant ;

- arrêter ce qui s'évacue trop abondamment ;

- rajouter ce qui manque.

Il devra aussi penser à jouer sur les qualités, c'est-à-dire à refroidir, réchauffer, dessécher, humecter, selon le cas.

Les traitements qui lui permettent d'obtenir ces résultats sont :

- les émétiques, laxatifs et diurétiques ;

- les massages, bains chauds, bains de vapeur, ventouses ;

- les saignées, cautérisation, incisions.

L'ensemble a sa cohérence, fondée à la fois sur une réelle observation et sur un raisonnement logiquement construit.

Chez Galien les progrès dans la connaissance de l'anatomie ont développé une médecine des organes qui va enrichir la médecine des humeurs, notamment avec la notion d'organes filtres comme le foie et la rate.

La médecine arabe médiévale a la même conception du corps et de la maladie :

6. Trois genres de corps constituent l'homme : les esprits, les fluides et les solides. Ses esprits ce sont les vapeurs qui l'habitent; ses fluides, ses humeurs qui sont le sang, le flegme, la bile jaune et l'atrabile. Ses solides : le cartilage, l'os, la chair et tout ce qui les environne comme la peau et autres choses semblables.

122. Si une quantité d'humeur, stationnant dans le sang, s'accroit dans des proportions qui dépassent la normale, la couleur $d u$ corps change, et l'homme contracte les maladies dues à cette humeur. $(11, p .51$, 81)
Cette médecine des humeurs a eu des conséquences importantes dans de nombreux domaines, et a largement débordé l'Antiquité et le Moyen Age.

En thérapie, c'est selon ses principes que l'on a pratiqué les saignées jusqu'au XVIIIè siècle, que l'on a utilisé les sangsues au XIXè (en telle quantité que tout pharmacien avait son bocal de sangsues en vitrine, que l'élevage en était florissant et le commerce international très important), que les ventouses jusqu'au milieu du XXè visaient à déplacer les humeurs pour décongestionner la partie du corps engorgée.

Nous avons gardé de cette conception du corps le vocabulaire courant des caractères, la personne chez laquelle une humeur prédominait étant imaginée se comporter selon cette dominance. Et l'on était, et l'on est toujours, sanguin, colérique ou bilieux, lymphatique ou flegmatique, mélancolique, de bonne ou de mauvaise "humeur".

Il se peut aussi que l'argumentation publicitaire des eaux minérales censées faire couler une eau pure à l'intérieur de notre corps, et ainsi le nettoyer de ses impuretés, relève du même imaginaire. De même que la pensée qui est à la base de l'emploi excessif de laxatifs dans notre société.

Scientifiquement, on parle toujours de milieu intérieur, et le programme du Premier Cycle des Etudes Médicales comporte toujours des chapitres sur "les compartiments liquidiens", "la régulation des volumes cellulaires" et "l'équilibre acidobasique".

\section{THEORIE DES HUMEURS ET REPRE- SENTATION DE LA SEXUALITE}

Le sperme est un liquide produit par le corps. Mais quelle est l'humeur qui peut en être l'origine? Assez vite va s'imposer la conviction que le sperme s'élabore à partir du sang, puisqu'il donne la vie comme lui. Savoir comment sera plus délicat : les 
observations sont multiples, mais il est difficile de les rendre cohérentes. Pour nourrir son argumentation contre la masturbation, Tissot va en 1760 recueillir toutes les références attestant "l'importance de la liqueur séminale". Le citer nous permettra un tour d'horizon instructif :

Pour Hippocrate, "la semence de l'homme vient de toutes les humeurs de son corps, elle en est la partie la plus importante. Ce qui le prouve, c'est la faiblesse qu'éprouvent ceux qui en perdent par l'union charnelle, quelque petite que soit la dose qu'ils en perdent. Il y a des veines et des nerfs qui de toutes les parties du corps vont se rendre aux parties génitales (...) Les humeurs entrent dans une espèce de fermentation qui en sépare ce qu'il y a de plus précieux et de plus balsamique, et cette partie, ainsi séparée du reste, est portée par la moelle épinière aux organes génitaux".

Pour Galien, "cette humeur n'est que la partie la plus subtile de toutes les autres, elle a ses veines et ses nerfs qui la portent de tout le corps aux testicules. En perdant la semence, on perd en même temps l'esprit vital ; ainsi il n'est point étonnant qu'un coït trop fréquent énerve, puisqu'il prive le corps de ce qu'il a de plus pur".

Pour Aristote, "c'est la partie la plus perfectionnée de nos aliments".

Pour Pythagore, "c'est la fleur du sang le plus pur".

Pour Alcméon, la semence "est une portion du cerveau, et il n'y a que quelques années qu'un médecin célèbre a adopté et amplifié ce système : il indique les passages par lesquels le cerveau va aux testicules, qu'il regarde comme des ganglions et non pas comme des glandes, et c'est par la dissipation du cerveau qu'il explique tous les phénomènes de l'épuisement vénérien".

Platon "envisageait cette liqueur comme un écoulement de la moelle de l'épine. Démocrite pensait comme Hippocrate et Galien. Epi- cure (...) regardait la semence comme une parcelle de l'âme et du corps, et fondait sur cette idée les préceptes qu'il donnait de la conserver soigneusement". [15, p. 71-73]

Ses contemporains ne sont pas négligés par Tissot :

M. de Gotter est dans la même idée : le sperme est la plus parfaite et la plus importante des liqueurs animales, la plus travaillée, le résultat de toutes les digestions; son intime rapport avec les esprits animaux prouve que, comme eux, elle tire son origine des humeurs les plus parfaites. [15, p. 74]

Lui-même précise qu'il y a des humeurs qui sont expulsées aussitôt individualisées (la sueur), d'autres collectées un certain temps avant l'expulsion (l'urine); tandis que d'autres sont : séparées et retenues dans des réservoirs pour (y) acquérir une perfection qui les rend propres à de nouvelles fonctions, quand elles rentrent dans la masse des humeurs. Telle est, entre plusieurs autres, la liqueur génitale. Séparée dans les testicules, elle passe de là par un canal assez long dans les vésicules séminales, et est constamment repompée par les vaisseaux absorbants, et, de proche en proche, rendue à la masse de toutes les humeurs. [15, p. 76$]$

Ce que prouverait l'absence de pollution chez des hommes continents pendant des années : sinon où serait passée toute la semence produite? La réabsorption est l'explication logique de l'action des productions testiculaires sur l'organisme. Ainsi, pour Galien :

La semence est gardée dans les vésicules séminales jusqu'à ce que l'homme en fasse usage ou que les écoulements nocturnes l'en privent (...) Mais la plus grande quantité de cette semence, la plus volatile, la plus odorante, celle qui a le plus de force, est repompée dans le sang, et elle y produit, en $y$ entrant des changements bien surprenants ; la barbe, les poils, les cornes; elle change la voix et les mours, car l'âge ne produit pas dans les animaux ces changements, c'est la 
semence seule qui les opère, et on ne les remarque jamais dans les eunuques. [15, $p$. 78]

Mais Tissot n'explique pas pourquoi le sperme est blanc alors que le sang est rouge. D'autres ont pensé que le passage dans le cerveau opérait ce changement de couleur, les veines conduisant du cerveau aux testicules y amenant du sang déjà blanchi qui servait à la nutrition du cerveau. D'autres, comme Galien, croient plutôt à la cuisson du sang dans les circuits testiculaires "innombrables comme les vrilles de la vigne". Ainsi Rhazès :

Aux testicules arrivent des rameaux se détachant des branches du corps ; ces rameaux, en s'entortillant, forment des circonvolutions nombreuses qu'entoure une chair glanduleuse blanche qui convertit le sang $q u$ 'elle contient, jusqu'à ce qu'il devienne blanc. $[14, p .8]$

Les circonvolutions des canaux séminifêres sont interprétées comme celles d'un alambic, et le blanchiment du sperme à celui du vin, dont la distillation donne un liquide blanc aussi, "l'esprit de vin", l'alcool, qui concentre les qualités du vin comme le sperme concentre les qualités vitales du sang. Henri de Mondeville, chirurgien de Philippe le Bel, écrira, comme plus tard Vésale, que :

Le superflu du bon sang nutritif de tous les membres est amené aux testicules. Quand il a subi une nouvelle digestion dans les testicules et les vaisseaux spermatiques, il forme la matière spermatique [9].

Une telle conception du sperme comme un des liquides contenus dans le corps humain va amener les mêmes comportements qu'envers les autres humeurs. Tout le problème sera ainsi de savoir garder l'équilibre entre évacuation du trop-plein et risque de pertes excessives.

Pour Rhazès "le sperme est une superfluité dont la longue stagnation dans le corps peut causer des maladies putrides. Aussi faut-il le rejeter modérément" [8, p. 92]. En 1623, le docteur Jacques Ferrand n'ose présenter la même suggestion, et propose plutôt d'attaquer le mal à sa source, en saignant le patient avant que le cerveau soit atteint :

$V u$ que la semence n'est qu'un sang blanchi par la chaleur naturelle et un excrément de la troisième digestion qui irrite par sa quantité la nature à le rejeter hors du corps : autrement il se corrompt dans ses réservoirs, et de là il jette au cerveau, par la moelle épinière et autres conduits occultes, mille vapeurs qui troublent ses facultés et vertus principales : il sera fort utile de tirer hors du corps la quantité superflue du sang par la saignée de la veine basilique du bras droit [4].

Au XVIIIè siècle Buffon confirme :

Le mariage, tel qu'il est établi chez les peuples raisonnables, est l'état dans lequel l'être humain doit faire usage des nouvelles facultés qu'il a acquises par la puberté, qui lui deviendraient à charge et même quelquefois funestes, s'il s'obstinait à garder le célibat. Le trop long séjour de la liqueur séminale dans ses réservoirs peut causer des maladies dans l'un et l'autre sexe, ou du moins des irritations très violentes [3, p.258].

A la Renaissance, Sylvius, partageant la conviction qu'il existe un sperme féminin, montre que le problème est le même pour les femmes :

Quand une femme, principalement celle qui est jeune et dynamique, bien en chair, bien nourrie et abondante en sang et en semence, est soit religieuse, soit volontairement chaste, soit mariée à un homme qui s'adonne peu à la femme, soit veuve d'un mari qui était fort sujet au plaisir, quand une telle femme est tentée du désir de Vénus, excitée par le regard de quelque homme, par une conversation impudique ou lascive, par des baisers ou des attouchements des tétins ou 
des parties naturelles, même si c'est seulement en songe, elle répand largement sa semence en la matrice. Cette semence se convertit en faux germe, ou se corrompt à l'intérieur de la matrice ou bien environ les génitoires, et élève au cour et au cerveau certaines vapeurs corrompues, desquelles s'engendrent de cruels accidents $[1, p .47]$.

Mais depuis l'Antiquité il existait des critiques de l'idée de sperme féminin, et, en tout cas, beaucoup refusaient à ce liquide produit par les femmes lors de l'excitation sexuelle la même qualité d'élaboration qu'au sperme mâle. D'ailleurs, il est plus aqueux, et les femmes n'ont pas l'équivalent de l'alambic testiculaire qui permettrait une vraie élaboration. C'est pourquoi :

L'influence du physique de l'amour paraît produire moins de ravage chez les femmes que chez les hommes. Il est facile de l'expliquer : la liqueur qu'elles répandent est moins précieuse, moins travaillée que celle des hommes. D'ailleurs une partie des femmes étant difficile à émouvoir, et une autre partie étant d'une constitution absolument inhabile au plaisir, les excès ne sont pas pour elles [7, t. II, p. 127].

Celles qui ont un tempérament ardent n'ont pas non plus à craindre les conséquences fâcheuses sur le cerveau d'éventuels excès puisque la nature, prévoyante, ne leur a donné ce tempérament que "pour les dédommager du peu d'esprit qu'elles ont" [7, t. II, p. 128]. La preuve en est que les femmes lubriques et qui savent raisonner font des enfants chétifs (leur force vitale, dépensée dans leur activité cérébrale et dans leurs plaisirs, manque pour donner une vie de qualité à leur enfant).

Le Grand Larousse Encyclopédique de 1870 attribuera lui aussi à la chasteté des femmes toutes sortes de maux, mais, semble-t-il, plus par l'absence de régénération apportée par la force vitale du sperme que par altération des humeurs féminines trop longuement stockées : "les filles céliba- taires ont une santé altérée et ressemblent à des fleurs pâles qui attendent les rayons fécondants de l'astre du jour. C'est principalement parmi elles que se rencontrent diverses affections de l'utérus, des squirres, des cancers à cette partie ou aux seins. Leur vie est plus courte ; car le célibat est moins favorable, en règle générale, à la longévité" (article Femme). Et la conviction s'installe que "toutes les maladies génitales des femmes célibataires ou veuves disparaissent par le mariage", et au milieu du XXè siècle des médecins témoigneront : "nous avons vu un grand nombre de jeunes filles chlorotiques, anémiques, atteintes de dysménorrhées, de névralgies, de migraines, de troubles divers de la circulation ou du système nerveux guéries par le mariage" [5, p. 96].

Si les uns ont peur des maux entrainés par la stagnation, en revanche, pour d'autres, ce sont les risques de pertes excessives qui peuvent obnubiler. Pythagore, au VIè siècle avant Jésus-Christ conseillait de pratiquer la sexualité en hiver plutôt qu'en été, et avec modération au printemps et en automne, tout en gardant à l'esprit que, quelle que soit la saison, cet exercice restait fondamentalement mauvais pour la santé. Hippocrate affirme qu'il convient de conserver sa semence afin d'insuffler au corps le maximum d'énergie et d'éviter, par une trop grande dépense de liqueur séminale, la tuberculose de la moelle épinière, voire la mort. Soranos prône la chasteté comme facteur de santé. Pour Démocrite le coït est une espèce d'épilepsie. Au Moyen Age, de Maïmonide à Arnaud de Villeneuve, l'abus de coït abrège toujours l'existence, et on se fait peur en répétant que l'affaiblissement dù à une dépense de liqueur spermatique correspond à celui que causerait la perte de quarante fois son volume de sang. En 1772, De Lignac Ecrit :

Autant le côté physique de l'amour, lorsqu'on en use avec modération répand des influences salutaires sur la santé, autant 
son usage excessif nous plonge dans des accidents funestes. L'importance de la liqueur séminale pour entretenir une santé vigoureuse exige qu'une partie de cette liqueur précieuse soit repompée dans la masse de sang après qu'elle a atteint toute sa perfection. Rien ne peut la remplacer en nous $[7, t . I I, p .112-113]$.

Et, afin "d'épouvanter les hommes téméraires qui sacrifient leur santé aux plaisirs", il cite l'exemple d'hommes et de femmes morts pour avoir eu des rapports à un moment où ils étaient déjà affaiblis, par une maladie ou une opération, et de jeunes mariés emportés en une nuit ou une semaine pour avoir oublié toute mesure : "épuiser ses forces par des jouissances trop répétées, c'est se creuser un précipice dont on ne s'apercevra que lorsque l'on y sera tombé". On dit bien des gros mangeurs qu'ils creusent leur tombe avec leurs dents...

Au XIXè siècle, Balzac s'applique le principe "aut libri, aut liberi", c'est-à-dire que quand il désire écrire des livres il ménage ses forces en n'ayant pas de rapport. Le sperme venant du cerveau ou participant à la vitalisation du cerveau, celui qui s'épuise dans les activités sexuelles entraîne un rétrécissement et même un dessèchement du cerveau, à tel point qu'on peut l'entendre "cogner contre les parois du crâne" : on ne peut donc accomplir des prouesses intellectuelles que si l'on n'a pas une activité sexuelle trop développée. Le Grand Larousse déjà cité constate que "rien n'énerve et n'épuise tant les forces qu'une éjaculation fréquente et l'excrétion d'une grande quantité de sperme". L'encyclopédie Bilz de médication naturelle, vers 1900 , insiste :

L'excès est un péché qui use trop tôt les meilleures sèves vitales ... La conséquence en est un défaut de chaleur et de forces vitales, d'où résulte une mauvaise digestion, la tristesse et une vieillesse prématurée, des maladies graves et une mort hâtive ... Seul le médecin expérimenté connaît les tristes causes de la faiblesse, de la difformité et des vices de conformation des descendants... [2, p. $517 \%$.

En 1932, une femme médecin écrit encore :

Il serait désirable que les jeunes épouses trop aimantes sachent que leurs maris se donnent plus qu'elles dans cette union, et que l'acte qui achève l'épanouissement de leur beauté et cause leur joie altère à la longue, lorsqu'il est trop souvent répété, la santé de ceux qu'elles aiment, car à chaque fois c'est un peu de leur influx nerveux et de leur vitalité qu'ils dépensent, ce qui fait que l'abus peut leur devenir funeste, ou causer leur vieillesse prématurée [5, p. 97].

Et un traité de sexologie de 1966 met en garde :

Pour la plupart, un rapport ou deux par semaine semble la normale. Des états d'épuisement, de nervosité, des douleurs abdominales basses et lombo-sacrées sont des indices d'excès. Une activité sexuelle excessive peut facilement déterminer un épuisement particulièrement dangereux pour le système nerveux $[6, p .160]$.

Donc, d'Hippocrate à nos jours, les mêmes imaginaires se manifestent, traduisant les mêmes peurs devant la sexualité. Ce qui va pousser certains groupes à des comportements extrêmes : afin d'empêcher toute déperdition de cette semence vitale, ils vont se castrer volontairement, estimant ainsi garder en eux le souffle vital, qui pourra se transformer en souffle psychique. A l'époque romaine, les Galles, adorateurs de Cybèle, savaient que la section du canal déférent "empêchait le départ du sang le plus pur vers le pénis pour la fécondation", et que, si l'appareil génital transmet "le souffle en fécondant la femme et en donnant naissance à l'enfant, (par la castration) le souffle peut se conserver, poursuivre son trajet pour devenir le souffle psychique, celui de l'homme supérieur" $[12$, p. 157, 164]. Au XIXè siècle, la secte russe des Skoptzy relancera la pratique de la cas- 
tration comme voie vers la perfection. Et de nos jours les castrats de l'Inde sont censés acquérir par leur amputation une qualité d'être qui les met en rapport avec la divinité, leur permettant de jeter des mauvais sorts ou au contraire de bénir les nouveaunés pour les protéger des coups du sort.

Une des conséquences contemporaines de cet imaginaire est l'attitude vis-à-vis de la sexualité des sportifs. Quand un corps doit produire un grand effort, aller au bout de ses possibilités, comment faut-il gérer son énergie vitale?

Platon disait, dans Les lois, que le champion olympique Issos de Tarente était ambitieux et "possédait au fond de son âme la technique et la force de la modération. C'est pourquoi, aussitôt entamée la période d'entraînement "il ne touchait plus ni une femme, ni un jeune garçon". Chez les Grecs, la même chasteté est exigée de leurs guerriers avant les combats. Mais les gladiateurs romains, qui passaient la nuit précédant leur passage dans le cirque en festins, avec des femmes, et qui pourtant montraient une grande ardeur au combat, commencent à faire douter les médecins. Pourtant au XXè siècle les entraîneurs interdiront aux sportifs la présence de partenaires dans les stages préparatoires et les compétitions, en athlétisme, en football, en cyclisme... Il a fallu que dans les années soixante-dix un athlète passe son temps entre les épreuves olympiques à courir les femmes tout en remportant plusieurs médailles d'or pour que la certitude s'effrite. Depuis, la partenaire habituelle (pas une nouvelle, qui pourrait stresser) a été acceptée auprès des footballeurs lors des deux dernières coupes du monde, auprès d'athlètes lors de certains stages... Mais les femmes des cyclistes ne peuvent toujours pas les accompagner lors du Tour de France.

\section{CONCLUSION}

Il ne s'agit pas de tourner en dérision les convictions d'autrefois, mais de comprendre comment le raisonnement a pu conduire nos prédécesseurs à se construire de telles certitudes et à en déduire de telles règles comportementales. Et ceci pour deux raisons. La première, que nous ne sommes pas les premiers à construire des raisonnements, et que toute la théorie des humeurs et ses conséquences sur la sexualité sont le fruit de raisonnements très élaborés. Comme le rappelle Claude Thomasset "on ne véhicule pas des absurdités pendant des siècles, mais tout simplement des réponses cohérentes aux questions fondamentales" [14, p. 17]. La deuxième, que nous ne sommes pas du tout sortis de cet imaginaire à propos du corps humain, comme de nombreuses citations de contemporains le montrent, alors que la science nous propose aujourd'hui de tout autres explications des phénomènes.

Rappelons que, afin de donner une "réponse cohérente à la question fondamentale" de l'effet sur le corps des hommes des produits testiculaires, la science contemporaine a rejeté la théorie du repompage d'une partie de la liqueur spermatique, en dissociant deux circuits : l'un hormonal, où la production testiculaire passe directement dans le sang, provoquant toutes les manifestations du caractère mâle ; l'autre spermatique, où la production testiculaire de spermatozoïdes rejoint celle de plusieurs autres glandes pour former le sperme, dans un circuit ouvert seulement sur l'extérieur. Le sperme est stocké sans pouvoir être réabsorbé, et sa seule destination est l'expulsion, volontaire ou non, les capacités de stockage étant limitées. Donc tous les discours sur la perte d'énergie ou de vitalité, et tous les comportements qui dérivent de la croyance en cette perte, toujours fréquents chez nos contemporains, sont fondés sur de purs fantasmes. Il serait plus logique aujourd'hui de concevoir le plaisir sexuel comme une décharge d'endorphine, et non comme une perte d'énergie, et d'y voir un stimulant cérébral, le rôle émotionnel, d'équilibre et de détente joué par l'orgasme étant indéniable [12]. Rufus d'Ephè- 
se disait déjà à la fin du premier siècle que l'acte sexuel "dissipe les idées fixes et adoucit les passions indomptables : voilà pourquoi il n'existe aucun remède aussi efficace que celui-là contre la mélancolie". Il est rejoint par Martin Le Maistre, recteur de l'Université de Paris à partir de 1464 . Ce célèbre professeur affirme en effet :

Je dis que chacun peut être autorisé à jouir de son plaisir, premièrement par pur goût $d u$ plaisir et deuxièmement pour échapper au dégoût de l'existence et aux tourments de la mélancolie qui accompagnent le manque de satisfaction des sens. S'il cherche à illuminer la tristesse qui s'installe en cas d'absence de jouissance sexuelle, le rapport conjugal n'est pas coupable [10, p. 276].

Etudier les théories anciennes, connaître l'histoire des idées, médicales ou populaires, sur des problèmes de santé et d'hygiène, permet de comprendre l'origine d'explications tellement efficaces, nourrissant si bien l'imaginaire, si bien fondées sur le vécu des individus, qu'elles sont toujours opérantes pour nous malgré des connaissances scientifiques théoriquement disponibles qui nous proposent des explications pourtant plus fines, compte tenu des découvertes effectuées.

\section{REFERENCES}

1. BERRIOT-SALVADORE E. : Un corps, un destin, La femme dans la médecine de la Renaissance. Paris, Champion, 1993.

2. BILZ F. E. : La nouvelle médication naturelle. Paris, Quillet, 1900.

3. BUFFON : Histoire naturelle de l'Homme. In Oeuvres complètes, tome IV. Paris, 1774.

4. FERRAND J. : De la Maladie d'Amour ou Mélancolie érotique. Paris, 1623.

5. GABORIAU H., GABORIAU A. : Les trois âges de la femme. Paris, Larousse, 1932.

6. HORNSTEIN F.-X., FALLER A., Sexologie. Salvator, 1966.

7. LIGNAC de : De l'Homme et de la Femme, considérés physiquement dans l'Etat du mariage. Lille, 1772.
8. MADELENAT P. Ed. : Etre femme et sportive. Paris, Doin, 1991.

9. MONDEVILLE H. de : Chirurgie, ch. Anatomie, IX. Traduction et Edition E. Nicaise, Paris, 1893.

10. RANKE U. : Des Eunuques pour le royaume des cieux. Hambourg, 1988. Paris, Laffont, 1990 et Hachette-Pluriel, 1992.

11. RAZI (ou RHAZES), Guide du médecin nomade, traduit par El-Arbi Moubachir. Paris, Sindbad, 1980 .

12. ROUSSELLE A. : Porneia. Paris, P.U.F., 1983.

13. SCHWOB M. : De l'amour plein la tête, ou la biologie de l'amour. Paris, Hachette, 1984.

14. THOMASSET C. : La représentation de la sexuali$t E$ et de la génération dans la pensée scientifique médiévale. In Love and Marriage in the twelfth century, Louvain, $1981: 1-17$.

15. TISSOT S.-A. : L'onanisme. 1760. Paris, La Différence, 1991.

\section{ABSTRACT}

\section{Theory of humours and sexuality}

\section{Y. Ferroul}

The old theory of humours has penetrated the minds in our society over the past two millennia to such an extent that it remains very much alive in spite of the emergence of new scientific theories. The main features of the theory of humours are summarized and the therapeutic principles that arose out of it are recalled. Sexuality is one of those domains in which the prescriptions engendered by the theory have been many, shaping imagination and behaviour. From Hippocrates onwards, doctors have advised and cared for their patients according to it, though it moralists have justified their judgements on sexual practices and it is from the image of the body that issues from it that many individual and social behaviours can be explained. The discovery of hormones and the modern explanation of the way the body works should have done away with the ideas and behaviours linked to the theory of humours : yet it still has such an impact on the imagination that it survives all scientific evidence.

Key words : theory of humours, sexuality, history of medicine, castration, sexuality and sport. 